\title{
Solution of Economic Load Dispatch (ELD) Problem by Path Relinking (PR) Algorithm
}

\author{
Christos Drosos \\ University of Piraeus \\ Dept. of Informatics \\ Piraeus, Greece
}

\author{
loannis Trichas \\ University of Piraeus \\ Dept. of Informatics \\ Piraeus, Greece
}

\author{
Aristidis Vlachos \\ Hellenic Merchant Navy \\ Academy of Aspropyrgos \\ Dept. of Mechanical \\ Engineering \\ Aspropyrgos, Greece
}

\begin{abstract}
This paper presents a solution of the Economic Load Dispatch (ELD) problem, using the Path Relinking Algorithm (PR). Generally, PR is a population-based meta-heuristic technique to integrate intensification and diversification strategies in a search procedure. Also, to explore trajectories that connect elite solutions .The economic load dispatch problem is formulated as the minimization of the production cost function, expressed as a quadratic polynomial, subject to the power balance constraints and to the generation limits constraints. The proposed approach has been applied in five generators units. The comparison with the real-coded genetic algorithm (RCGAs), the binary-coded genetic algorithm (BCGAs) and the classical optimization technique of QuasiNewton, demonstrates the superiority of the PR algorithm and confirms its potential to solve the ELD problem.
\end{abstract}

\section{General Terms}

Mathematical programming; numerical optimization; metaheuristics algorithms;

\section{Keywords}

Economic Load Dispatch Problem, meta-heuristic optimization, Path Relinking algorithm.

\section{INTRODUCTION}

The Economic Load Dispatch (ELD) problem is one of key problems in power operation and planning. The ELD problem may be expressed by minimizing the total generation cost while satisfying an equality constraint and several inequality constraints.

The ELD problems have complex and nonlinear characteristics. To solve these problems, various algorithms and heuristic approaches have suggested or investigated by power engineers, including Lagrangian relaxation [1], gradient method [2], linear programming and dynamic programming [3], interior point method [4]. More recently, heuristic techniques, such as genetic algorithms [5][6], simulated annealing [7], evolutionary computing [8], PSO algorithms [9][10] , tabu-search [11] and Ant Colony[12] have also been intensively investigated. In this paper, Path Relinking algorithm which was proposed by Glover [13][14] introduced.

The rest of the paper is organized as follows:

In section 2, the principal framework of the Path Relinking algorithm is reviewed. In section 3, is presented the formulation of economic load dispatch problem. In section 4, is presented the PR algorithm for ELD problem and in section the case studies. Finally, a conclusion is given in section 6 .

\section{THE PATH RELINKING ALGORITHM}

Path Relinking is a meta-heuristic technique, proposed by Glover which has been suggested as an approach to integrate intensification and diversification strategies in a search procedure [14].

The PR algorithm operates on a set of elite (high-quality) solutions, called Reference set (Refset). Starting from one of these solutions, called an initial solution, the aim of the search is to generating a path, by performing moves, in neighbourhood space (Figure 1) that leads toward the other solutions, called guiding solutions [16].

Therefore, new better solutions may occur along the path.

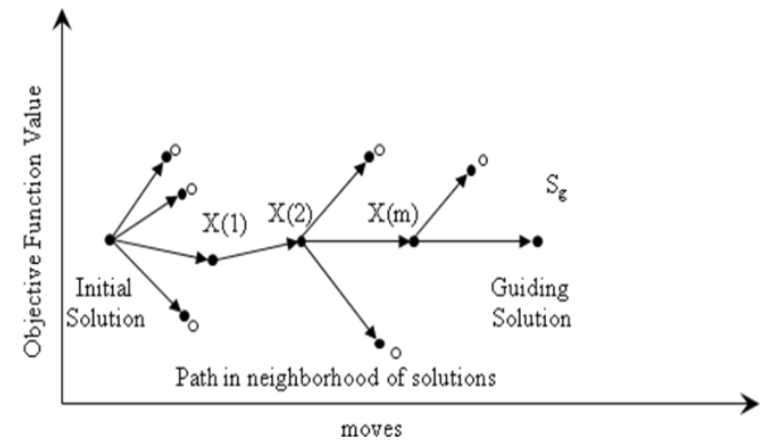

Figure 1 The process flow of the PR algorithm generates new solutions by exploring trajectories that connect elite solutions.

For the intermediate solutions: $x(1), x(2), \ldots, x(n)$ of the path is possible to introduce the meaning of distance between the solutions and define a neighbourhood of a solution as a whole of all the solutions, where distance from the current solution cannot exceed a certain threshold.

In the case that a solution can be expressed from binary vectors (the vectors are constrained to take values 0 or 1 ) with $\mathrm{N}$ components, it can be used the Hamming distance. The Hamming distance between two solutions $\mathrm{x}$ and $\mathrm{x}^{\prime}$ which computed by:

$$
d_{H}\left(x, x^{\prime}\right)=\sum_{j=1}^{n}\left|x(j)-x^{\prime}(j)\right|
$$

Generally, the PR algorithm requires the following, [14]:

- A neighborhood structure for the movements. 
- Selection criteria for the path.

- Selection criteria for intermediate solutions of the path.

- $\quad$ Selection criteria for initiating (Si) and guiding $(\mathrm{Sg})$ solutions.

The Basic mechanism of PR algorithm problem is given in Figure 2.

1. Generate an initial reference set of solutions (RefSet) of b quality solutions

2. Evaluate the solutions in RefSet and order them according to their objective function

3. Repeat

Select the initial and the guiding solutions

For each pair of solutions (subset)

Create new better solutions ( $\mathrm{x}$ *) that

improve the result

Check new solutions according the objective function

If $f\left(x^{*}\right)<f(x), \forall x \in$ RefSet, where $\mathrm{f}$ is the objective function, then,

Update and reorder the RefSet, End If

End For

Until a fixed number of iterations or a given amount of execution time is performed

4. Print the best solution

Figure 2 : Basic mechanism of PR algorithm

\section{FORMULATION PROBLEM}

The aim of the economic load dispatch (ELD) problem is to minimize the objective cost function of the production units, (equation 2), under the power balance equality constraint, (equation 3), and the production limits inequality constraint of the generators, (equation 4). The mathematical form of the ELD problem is [21]:

$$
\begin{array}{r}
\operatorname{MinF}_{T}(P)=\sum_{i=1}^{N} F_{i}\left(P_{i}\right) \\
\sum_{i=1}^{N} P_{i}=P_{D}+P_{L}
\end{array}
$$

$$
P_{i \min } \leq P_{i} \leq P_{i \max }
$$

Where:

$\mathrm{N}$ : is the number of running (on line) power units.

$P_{i}$ : is the power output of the $\mathrm{i}^{\text {th }}$ unit in MW.

$P:$ is the vector that contains all the $P_{i}$.

$F_{i}\left(P_{i}\right)$ : is the production cost currency units per hour for generator $i$.

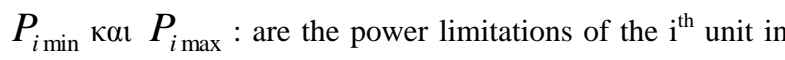
MW.

$P_{D}$ : is the total load demand in MW.

$P_{L}:$ transmission losses in MW.

In equation (1), the production cost function $F_{i}\left(P_{i}\right)$ is usually expressed as a quadratic polynomial:

$$
F_{i}\left(P_{i}\right)=\alpha_{i}+b_{i}+c_{i} P_{i}^{2}
$$

where $\alpha_{i}, b_{i}, c_{i}$ are cost coefficients of generator i.

Transmission losses are computed by:

$$
P_{L}=\sum_{i=1}^{N} \sum_{j=1}^{N} P_{i} B_{i j} P_{j}+\sum_{i=1}^{N} B_{0 i}+P_{i}+B_{00}
$$

where:

B: coefficients of transmission losses.

\section{ECONOMIC LOAD DISPATCH PROBLEM WITH PATH RELINKING (PR) ALGORITHM}

In this section describes the steps of PR algorithm in order to solve the ELD problem. The steps that the algorithm follows are:

Step 1: Initialization: Create a RefSet of solutions at random while satisfying constraints: equality, eq. (3) and inequality, eq.(4).

Step 2: Calculate the objective function, eq.(5) and order the solutions according to the value of the objective function.

Step3: Generate NewSubsetsCreation, which consists of new solutions resulting from the combination of the existing solutions.

Step4: If the new solution contributes to the optimization of the objective function, insert them in to the RefSet and reorder the solutions.

Step 5: Return at step 3 until the procedure is applied for all generators.

Step 6: If all iterations of the algorithm are completed, print the best solution for the problem.

After the initialization step, follows the creation of new solutions, using the space in the neighborhood of the already examined solutions. From the available solutions of RefSet, all the solutions are combined in pairs which include at least one new solution and they insert in a table with name "NewSubset". Then, while there are available SubSets for examination, they selected one by one and create the Initial and Guiding solution, then the Relinking method [22] applied to create pathways between these solutions. Each new solution after being examined for suitability and if its contribute to the optimization of the objective function, is imported to RefSet. Afterwards, the pair of solutions that was examined is removed from table NewSubset and the next pair of solutions from the table is selected for examination and search for a better path. After finish processing all table contents all current solutions have been combined and the PR 
algorithm proceeds to the next iteration, after returning the total produced cost value.

\section{CASE STUDIES}

The efficiency and effectiveness of the proposed PR algorithm has been applied to ELD problem with the generators [21]. The cost functions in dollars per hour as follows:

$F_{1}\left(P_{1}\right)=0,0015 P_{1}^{2}+1,8 P_{1}+40$

$F_{2}\left(P_{2}\right)=0,0030 P_{2}^{2}+1,7 P_{2}+60$

$F_{3}\left(P_{3}\right)=0,0012 P_{3}^{2}+2,1 P_{3}+100$

$F_{4}\left(P_{4}\right)=0,0080 P_{4}^{2}+2,0 P_{4}+25$

$F_{5}\left(P_{5}\right)=0,0010 P_{5}^{2}+1,9 P_{5}+120$

Power generators limits:

$100 \leq P_{1} \leq 300$

$80 \leq P_{2} \leq 150$

$80 \leq P_{3} \leq 200$

$20 \leq P_{4} \leq 100$

$100 \leq P_{5} \leq 300$

The total load demand, $P_{D}$, was 730 MW. Transmission losses $P_{L}$ are computed using the B coefficients.

The proposed algorithm was implemented in Matlab 7.0 with $\mathrm{CPU}$ i3 at $3.70 \mathrm{GHz}$, RAM 8GB and operating system Windows 10.

The value of the objective function, which calculates the total cost operation of 5 generators, is given from equation (2) and is calculated at the end of each iteration.

For the initialization and the specification of RefSet a population of $\mathrm{N}$ elements is considered. Each one of the elements represents a set of power values ( $P_{1}, P_{2}, P_{3}, P_{4}, P_{5}$ ) which could be considered as an arbitrary trial solution of the problem in study. After the initialization, the solutions are ordered according to the value of the objective function. The first solution corresponds to the best solution which is determined as the best produced power for each one of the 5 generators according to the minimum cost operation.

Figure 3 shows the value of total fuel cost $(\$ / \mathrm{h})$ as a function of the iteration number.

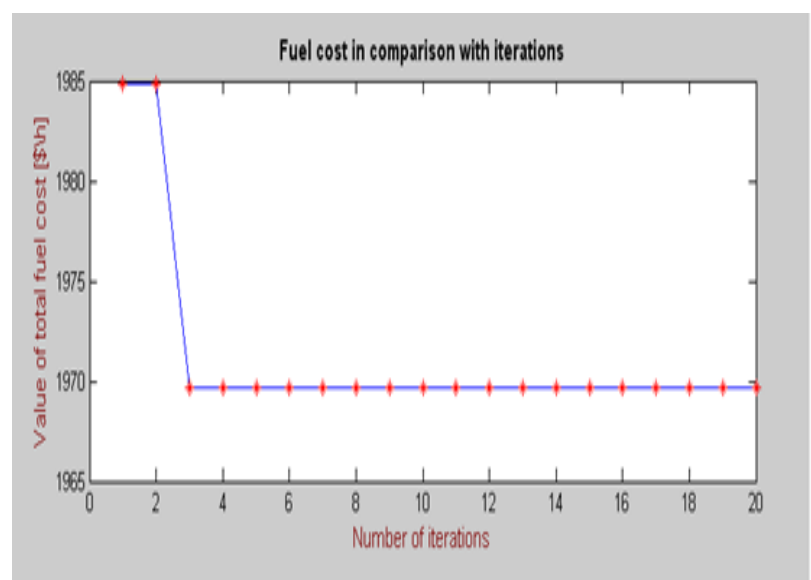

Figure 3 : Finding the lowest total fuel cost
Specifically, at twenty iterations, observed the following results for the total fuel cost of generators (Table 1).

Table 1 Results of the PR algorithm

\begin{tabular}{|l|l|l|}
\hline Generator i & $\begin{array}{l}\text { Active power } \\
\text { generations in MW }\end{array}$ & Cost $(\$ / \mathrm{h})$ \\
\hline 1 & 151.00 & 346.001500 \\
\hline 2 & 118.571262 & 303.748578 \\
\hline 3 & 189.194436 & 540.261758 \\
\hline 4 & 100.00 & 305.00 \\
\hline 5 & 171.234301 & 474.666359 \\
\hline total & 730.00 & 1969.678195 \\
\hline
\end{tabular}

The minimum total fuel cost and active power generations are presented in table 2 .

Table 2 : Total fuel cost of generators for iteration.

\begin{tabular}{|l|l|}
\hline Iterations & Total Fuel Cost \\
\hline $1^{\eta}$ & $1984,90 € / \mathrm{h}$ \\
\hline $2^{\eta}$ & $1969,702046 € / \mathrm{h}$ \\
\hline $3^{\eta}$ & $1969,678195 € / \mathrm{h}$ \\
\hline $4^{\eta}$ & $1969,678195 € / \mathrm{h}$ \\
\hline $5^{\eta}$ & $1969,678195 € / \mathrm{h}$ \\
\hline $6^{\eta}$ & $1969,678195 € / \mathrm{h}$ \\
\hline $7^{\eta}$ & $1969,678195 € / \mathrm{h}$ \\
\hline $8^{\eta}$ & $1969,678195 € / \mathrm{h}$ \\
\hline $9^{\eta}$ & $1969,678195 € / \mathrm{h}$ \\
\hline $10^{\eta}$ & $1969,678195 € / \mathrm{h}$ \\
\hline $11^{\eta}$ & $1969,678195 € / \mathrm{h}$ \\
\hline $12^{\eta}$ & $1969,678195 € / \mathrm{h}$ \\
\hline $13^{\eta}$ & $1969,678195 € / \mathrm{h}$ \\
\hline $14^{\eta}$ & $1969,678195 € / \mathrm{h}$ \\
\hline $15^{\eta}$ & $1969,678195 € / \mathrm{h}$ \\
\hline $16^{\eta}$ & $1969,678195 € / \mathrm{h}$ \\
\hline $17^{\eta}$ & $1969,678195 € / \mathrm{h}$ \\
\hline $18^{\eta}$ & $1969,678195 € / \mathrm{h}$ \\
\hline $19^{\eta}$ & \\
\hline $20^{\eta}$ & \\
\hline &
\end{tabular}

In the table 3 , the results of PR algorithm are compared with results of RCGAS, BCGAS and BFGS methods. 
Table 3 : Results of PR algorithm compared with RCGAS, BCGAS and BFGS methods.

\begin{tabular}{|c|c|c|c|c|}
\hline & RCGAS & BCGAS & BFGS & PR \\
\hline $\begin{array}{l}\mathrm{P}_{1}^{\mathrm{opt}} \\
(\mathrm{MW})\end{array}$ & 213.68 & 206.72 & 211.30 & 151.00 \\
\hline $\begin{array}{l}\mathrm{P}_{2}^{\mathrm{opt}} \\
(\mathrm{MW})\end{array}$ & 127.46 & 121.64 & 126.30 & 118.57 \\
\hline $\begin{array}{l}\mathrm{P}_{3}^{\mathrm{opt}} \\
(\mathrm{MW})\end{array}$ & 141.93 & 151.82 & 151.29 & 189.194436 \\
\hline $\begin{array}{l}\mathrm{P}_{4}^{\mathrm{opt}} \\
(\mathrm{MW})\end{array}$ & 29.53 & 33.21 & 71.24 & 100.00 \\
\hline $\begin{array}{l}\mathrm{P}_{5}^{\mathrm{opt}} \\
(\mathrm{MW})\end{array}$ & 258.86 & 258.05 & 211.31 & 171.234302 \\
\hline $\operatorname{Cost}(\$ / h)$ & 2010.8 & 2011.0 & 2029.3 & 1969.678195 \\
\hline
\end{tabular}

\section{CONCLUSION}

In this paper the Economic Load Dispatch (ELD) problem was studied and presented. In the literature, some heuristic approaches have been developed for deriving quality solutions to the ELD problem.

In this paper, the Path Relinking (PR) algorithm, a metaheuristic technique was used, as an approach to integrate intensification and diversification strategies in a search procedure.

Computational results have proved that the PR algorithm not only yields solution values that are comparable to those of RCGAS, BCGAS and BFGS algorithms but also gives better results.

\section{REFERENCES}

[1] Guan.X.Lux, P.B.Zhang L., Non Linear approximation method in Lagrangian relaxion based algorithms for hydrothermal scheduling, IEEE Trans Power Systems, vol.10, (2) (1995, PP: 772-778).

[2] Wood A.J Woolenberg B.F, Power Generation Operation and Control, John Wilex and sons, New York 1996.

[3] Chebbo A.M., Irving M.R., Combined active and reactive dispatch, Proc. IEE, Pt.c , (4), PP:393-405.

[4] Granville S., Optimal reactive dispatch through interior point methods, IEEE summer meeting, Paper No.92 SM 416-8 PWRS

[5] K.P.Wong and Y.W.Wong, Genetic and genetic/simulated annealing approaches to economic dispatch , IEE Proc. Gen. Trans. And Distrib,1994,141,(5),PP: 507-513

[6] Kaur A., Singh P. H. Bhardwaj, Analysis of Economic Load Dispatch Using Genetic Algorithm , International Journal of Application or Innovation in Engineering \& Management (IJAIEM), Volume 3, Issue 3, March 2014

[7] K.P.Wong and C.C.Fung, Simulated annealing based economic dispatch algorithm, IEE Proc.Gen.Trans. and Distirb. 1993,140,(6), PP:509-515.

[8] Hawkan A.H., Laughton M.A., Constraint governed genetic algorithm, (1996), Proc. $12^{\text {th }}$ PSCC.
[9] Vlachos A., Particle Swarm Optimization (PSO) techniques solving Economic Load Dispatch (ELD) Problem,Journal of Statistics and Management Systems ,Vol. 11, Iss. 4, 2008

[10] Abido, M. A., 2009. "Multiobjective particle swarm optimization for environmental/economic dispatch problem", Electric Power Systems Research, Vol. 79, No. 7, pp. 1105-1113.

[11] W.M.Lin.,F.S. Cheng M.T.Tsay, An improved Tabu Search for economic dispatch with multiple minima, IEEE Trans. On Power Systems ,Vol.17.No.1. PP:108112.2002

[12] Vlachos A., Petikas I. , Kyriakides S., A Continuous Ant Colony (C-ANT) algorithm solving the Economic Load Dispatch (ELD) Problem ,Journal of Information and Optimization Sciences ,Vol. 32, Iss. 1, 2011

[13] D.Corne , M.Dorigo and F. Glover (Editors), Now ideas in Optimization, London,McGraw Hill .1999.

[14] Glover, F., Laguna, M., Martí, R.: Scatter search and path relinking: Foundations and advanced designs. New Optimization Techniques in Engineering. Studies in Fuzziness and Soft Computing, vol. 141, pp. 87-100. Springer, Berlin (2004)

[15] Glover, F: Tabu Search and adaptive memory programming-advances, applications and challenges in: Interfaces in Computer Science and Operations Research , PP:1-75. Kluwer Academic Publishers, Dordrecht (1996)

[16] Glover F, Corne D, Dorigo M, Scatter search and path relinking,. New Ideas in Optimization ,1999, McGrawHill, Maidenhead, UK, PP:297-316

[17] Ho S, Gendreau M. Path relinking for the vehicle routing problem., Journal of Heuristics , 2006, Volume 12, Issue 1, PP: 55-72

[18] L.de O.Bostos and L.S.Ochi, A genetic algorithm with evolutionary Path-Relinking for the SONET Ring. Assignment Problem, Ing Opt 2008-International Conference on Engineering, optimization, Rio de janeiro,Brasil,01-05 June 2008

[19] R.P.Beansoleil Multiple Criteria Scatter Search.MIC 2001-4 ${ }^{\text {th }}$ Meta- heuristics International conferences, Porto, Portugal, July 16-20,2001.

[20] J.M.Pasia,H.Aguirre and K.Tanaka, Path Relinking on Many-Objective NK-landscapes.LNCS,2011,Volume 6238/2011,PP: 677-686.

[21] H.Bouzeboudja,A.Chaker,A.Alloli and B.Naama, Economic Dispatch solution using a real-coded genetic algorithm, Acta Electrotechnica et Informatica,No.4,Vol.5,P.P:1-5. 2005

[22] Glover,F, M.Laguna and R.Marti, Fundamentals of Scatter search and Path Relinking, Control and Cybemetics, 29(3),PP:653-684, (2002)

[23] Celso C. Ribeiro and Mauricio G. C. Resende , Pathrelinking intensification methods for stochastic local search algorithms ,Journal of Heuristics, 2012, Volume 18, Number 2, Page 193-214 